\title{
Intracellular signal transduction pathways as potential drug targets for ischemia-reperfusion injury in lung transplantation
}

\author{
Lei Huang ${ }^{1,2}$, Chengliang Yang ${ }^{1,2}$, Mingyao Liu ${ }^{1,2}$ \\ ${ }^{1}$ Latner Thoracic Surgery Research Laboratories, Toronto General Hospital Research Institute, University Health Network, Toronto, Canada; \\ ${ }^{2}$ Institute of Medical Science, Departments of Surgery, Medicine and Physiology, Faculty of Medicine, University of Toronto, Toronto, Canada \\ Correspondence to: Mingyao Liu, MD, MSc. James and Mary Davie Chair in Lung Injury, Repair and Regeneration, Professor of Surgery, Medicine \\ and Physiology, Faculty of Medicine, University of Toronto, Toronto, Canada. Email: mingyao.liu@utoronto.ca. \\ Comment on: Magruder JT, Grimm JC, Crawford TC, et al. Imatinib Is Protective Against Ischemia-Reperfusion Injury in an Ex Vivo Rabbit Model \\ of Lung Injury. Ann Thorac Surg 2018;105:950-6.
}

Submitted Aug 24, 2018. Accepted for publication Sep 19, 2018.

doi: 10.21037/jtd.2018.09.130

View this article at: http://dx.doi.org/10.21037/jtd.2018.09.130

\section{Lung transplantation and ischemia-reperfusion induced lung injury}

Lung transplantation is the primary life-saving treatment option for patients with end stage lung disease. However, about $15-20 \%$ of donor lung recipients in the posttransplant period develop primary graft dysfunction, a severe form of ischemia/reperfusion (IR)-induced lung injury, which is a major cause of morbidity and mortality in the first year after transplantation. Although many studies have been done to prevent and ameliorate IRinduced lung injury, the lack of effective drugs is still the main obstacle. Some of drugs have shown promising therapeutic effects in small animal studies, but they cannot be utilized clinically due to the lack of proper formulation. Some drugs have failed in clinical trials due to the big gap between small animals and humans, in terms of physiology, pharmacokinetics, drug efficacy, metabolism and potential toxicology. New drug targets should be explored, effective drug formulations must be developed, and drug delivery and testing strategies should be reconsidered.

\section{Imatinib in IR-induced lung injury—an "old" drug for "new" usage}

Recently, a paper entitled, "Imatinib is protective against ischemia-reperfusion injury in an ex vivo rabbit model of lung injury" was published by Magruder and co-workers at the Annals of Thoracic Surgery (1). In this report, Imatinib, a protein tyrosine kinase inhibitor (TKI), currently used to treat cancer, improved rabbit lung function during ex vivo lung perfusion (EVLP) (1). This is not the first study testing for Imatinib efficacy in IR-induced lung injury. However, this report along with other similar studies, has raised several interesting points in regard to drug discovery and delivery to treat the donor lung injury and to prevent or reduce ischemia-reperfusion induced acute lung injury during perioperative period of lung transplantation. First, repurpose current clinically applicable drugs is an important option for drug discovery. Second, Imatinib is a TKI that inhibits c-Abl, a non-receptor PTK, which supports the concept that intracellular signal transduction pathways could be targets for drug discovery in lung transplantation/acute lung injury. Herein, drug screening with bioinformatics, drug formulation and development of a drug-testing pipeline are discussed.

\section{Imatinib-repurpose from cancer to IR injury treatment}

Repurposing clinically applicable drugs for a new target is an important strategy in drug discovery. The safety ranges, potential side effects and contraindications in humans are better known. The underlying molecular mechanisms of the drugs may help to explain the therapeutic effects and adverse side effects seen in new clinical settings. Imatinib is used as an example to further elaborate this concept. BcrAbl tyrosine-kinase inhibitors are well known as a group of therapeutic drugs that were developed for patients with Bcr- 
Abl-positive chronic myelogenous leukemia (CML). They have also been proven to be able to treat gastrointestinal stromal tumors and other types of malignant tumors. Their major targets include Abl, Abl-related gene (Arg), plateletderived growth factor receptors, cKit and discoidin domain receptor tyrosine kinase 1 (2). Imatinib, a 2-phenyl amino pyrimidine derivative, is a representative of these drugs.

Recently, researchers have shown an increasing interest in the protective effect of TKI on IR-induced injury in different organs. Bcr-Abl TKI may play a pivotal role for being an anti-inflammation and attenuation of vascular permeability tool. Loss of Abl kinase activity was accompanied by activation of the barrier-stabilizing GTPases, Rac1 and Rap1 (3). Imatinib demonstrated itself as an effective prevention method of endothelial barrier dysfunction, and edema formation via inhibition of $\operatorname{Arg}$ (4). Through activation of PKGI, inhibition of c-Abl, increases key antioxidant enzymes and resistance to lung endothelial oxidant injury (5). In liver ischemia reperfusion, Nilotinib (a second generation of Imatinib) was reported to attenuate JNK phosphorylation, and hepatocellular apoptosis, and to block activation of p38 MAPK and cytokine production (6).

In the lung transplant setting, another example of repurposing drugs for IR-induced lung injury is the usage of alpha 1 anti-trypsin (A1AT), a drug which is used to treat emphysema patients with A1AT deficiency. It has been tested as a potential therapeutic for IR-induced lung injury in a cell culture model, rat lung transplantation model, pig lung transplantation model, and pig EVLP model (7). Based on these, and other studies, A1AT has been proposed for the use in clinical organ transplantation (8). In the future, we will see more drugs being repurposed for IR injury in lung transplantation.

\section{Other imatinib related drugs for IR injury}

Imatinib, as the first Bcr-Abl TKI on the market, was approved by FDA in 2001. Shortly after, some patients had experienced resistance and intolerance to imatinib (9). The second generation of drugs, which were developed to combat these limitations, included nilotinib, dasatinib, bosutinib, ponatinib. these drugs are approved for the treatment of imatinib-resistant or imatinib-intolerant CML. Some or even all of these four drugs, may replace Imatinib as a frontline standard TKI in the future due to their encouraging clinical data. Whether these new drugs can be used for IR-induced lung injury in transplantation is an interesting question.

\section{Other TKI and protein kinase inhibitors}

As is well known, non-receptor tyrosine kinases have been considered a primary mediator of intracellular signal transduction molecules and may have broad functions. In addition to c-Abl, other non-receptor tyrosine kinases may also be drug targets for IR-induced injury. Src protein tyrosine kinase (PTK) family is categorized in the nonreceptor tyrosine kinases category, which consists of nine members (10). Src PTK inhibition presents a new prophylactic treatment for pulmonary IR-induced acute lung injury. Src PTK phosphorylation (activation) was increased in ischemic donor lungs (11). Src PTK inhibitor (PP2) pretreatment in rats decreased phosphorylation of Src PTKs, total protein tyrosine phosphorylation, and STAT3 phosphorylation. PP2 reduced lung leukocyte infiltration, apoptotic cell death, fibrin deposition, and acute lung injury after reperfusion. Src inhibition also significantly reduced CXCL1 (GRO/KI) and CCL2 (MCP-1) chemokine levels in the serum (12).

In addition to the Src PTK and the c-Abl families, the roles of other protein kinases should also be explored. Protein kinase $\mathrm{C}$ (PKC) is a family of serine/threonine kinases, which also plays an important role in the intracellular signal transduction. Delta isoform of PKC (PKC delta) is activated during the cold ischemic time (CIT) in the donor lung preservation used with rats. Deregulated it via small interference RNA (siRNA) reduced IR-induced cytokine production and apoptotic cell death. The PKC delta inhibitor Delta V1-1, inhibited the translocation of PKC delta and p53 to the mitochondria after CIT, rescued ER stress protein expression, and converted the major mode of cell death from necrosis to apoptosis, thus effectively reduced lung IR injury in rats (13).

Activation of mitogen-activated protein kinases (MAPK) has also been observed in human lung transplants (14). The roles of these signaling pathways like, PTK, PKC, MAPK, and etc. should be explored in the lung transplant setting.

\section{Signal transduction as potential targets: bioinformatics \& high-throughput screening}

The past decade has seen the rapid development of bioinformatics, such as genomics, epigenetics, transcriptomics, proteomics and metabolomics. Data from these studies have contributed significantly to signal transduction-based drug discovery. Some of these technologies, tools and insights, have been used in the lung 


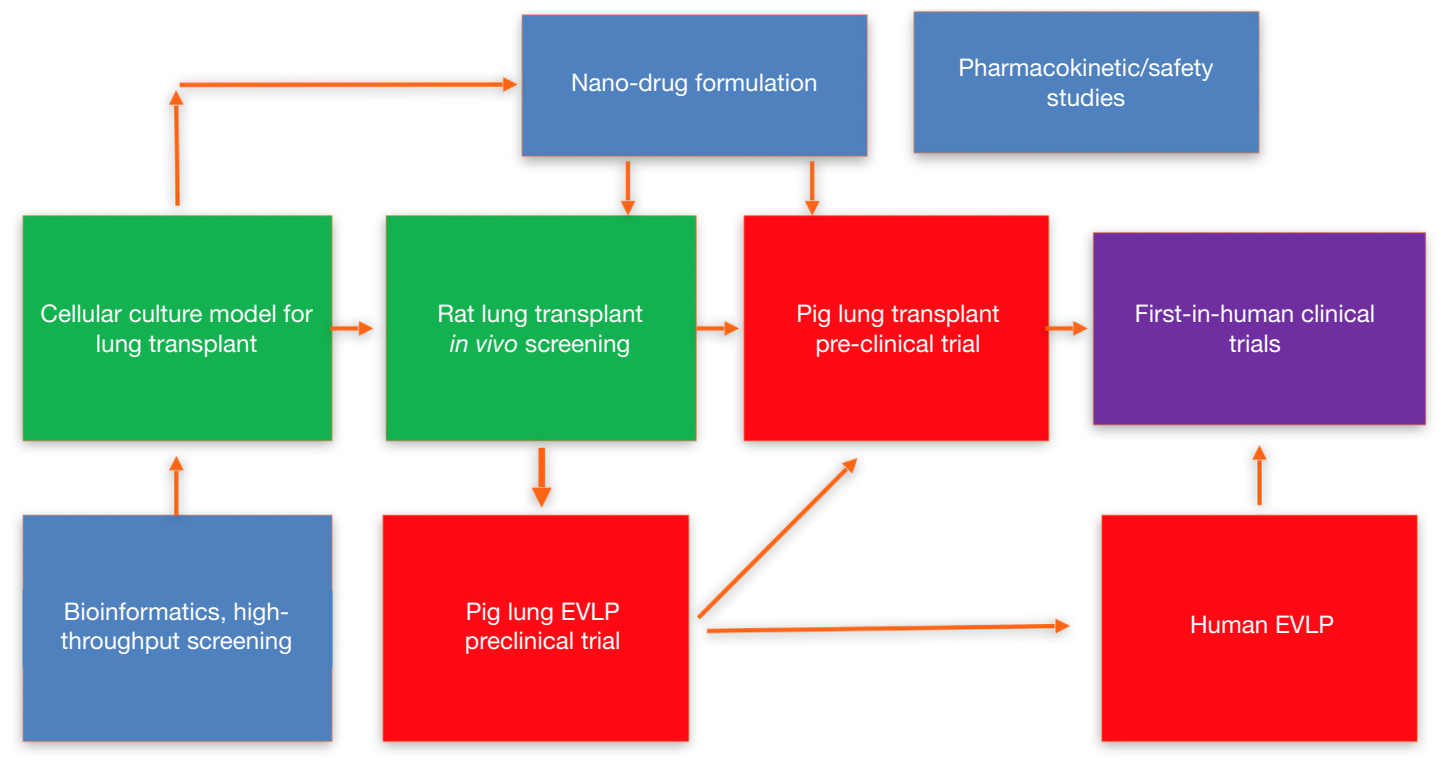

Figure 1 A proposed drug discovery and delivery pipeline for lung transplantation. EVLP, ex vivo lung perfusion.

transplant setting (15-18). However, most of these previous studies have not focused on drug discoveries. To design "omics" studies properly, with newly developed tools, such as DrugBank (19), it is possible to systemically search for drug targets in the IR process.

High-throughput screening is another commonly used technique in drug discovery. This method, due to utilization of robots, detectors and developed software that can automate the whole process, enables a series of analyses of chemical compounds to be conducted in a short time. Using it to validate the drug targets that were discovered from bioinformatics studies is a very attractive approach. The critical step in this process would be to develop cell culture models that can simulate the IR process in lung transplant (13).

\section{Formulation of drugs: hydrophobicity, intracellular delivery}

With the discovery of new drugs, the next challenge is how we can deliver them to patients. For example, many chemical compounds are hydrophobic (i.e., waterinsoluble), and some drugs have difficulty crossing the cell membrane. Drug formulation and drug delivery methods must be considered. Nanotechnology is an attractive tool for this purpose. Nanoparticles (NPs) can improve the drugs' pharmacokinetic, efficacy, safety, and targeting. For example, the Src PTK inhibitor PP2 is a hydrophobic chemical. Using self-assembly peptides and some amino acids, it has been formulated as an intravenously injectable drug, and used to reduce LPS-induced, acute lung injury in mice and IR-induced lung injury in rats $(20,21)$.

Another example is the PKC delta inhibitor, delta V1-1, as mentioned above. As a peptide-based drug, crossing the cellular membrane is the biggest challenge. In the past, a cell penetration peptide (TAT) has been used as a delivery vehicle (13); however, non-specific biological effects have been noted for TAT (22). The gold NP-peptide hybrids have showed a good stability level with high uptake (23). When formulated with delta V1-1, the GNP-peptide hybrid reduced IR-induced cell injury in vitro and pulmonary IR injury in vivo (24). In transplant settings, ex vivo organ perfusion provides a new route to repair injury of isolated organs. Using gold NP formulated drugs, may repair donor lung injury prior to lung transplantation.

\section{Drug discovery pipeline}

Another challenge for translating the drugs from lab testing to clinical application is that most of the initial studies in lung transplant are based on small animals, which differ greatly from human, in terms of physiology, pharmacokinetics, drug metabolism, etc. To successfully translate experimental findings to clinical applications, a drug discovery and delivery pipeline for IR injury in lung transplantation should be developed (Figure 1). Bioinformatics may provide multiple potential drug targets. 
A cell culture based high-throughput screening and testing models should be developed for mechanistic studies. These models should simulate major features of hypothermic preservation and normothermic reperfusion processes, which is typically involved in lung transplantation. Next, to determine the therapeutic effects of selected drugs or formula in vivo, small animal screening systems should be available, such as rat pulmonary ischemia-reperfusion models (12), or rat lung transplant models (13). To translate these findings to clinical trials, a pre-clinical pig lung transplant model should be established (25). Furthermore, EVLP technology allows to assess donor lung function and enables the repair of injured donor lungs. Using EVLP to treat damaged human donor lungs, provides invaluable information to support future clinical trials. Drug formulation, pharmacokinetics and toxicology studies are also required. A multi-disciplinary research team and interlaboratory collaborations are absolutely essential.

In summary, to prevent and treat IR injury in lung transplantation, intracellular signal transduction pathways are important therapeutic targets. Clinical drugs, such as Imatinib, can be repurposed, and new drugs can be discovered through bioinformatics and high-throughput screening. A drug discovery and delivery pipeline has emerged as a result of the scientific collaborations and integration of multi research groups.

\section{Acknowledgements}

Funding: This work is supported by Canadian Institutes of Health Research, operating grants MOP-42546 and PJT148847, and Ontario Research Fund (RE-08-029) from Ontario Government. Mingyao Liu is James \& Mary Davie Chair in Lung Injury, Repair and Regeneration.

\section{Footnote}

Conflicts of Interest: The authors have no conflicts of interest to declare.

\section{References}

1. Magruder JT, Grimm JC, Crawford TC, et al. Imatinib Is Protective Against Ischemia-Reperfusion Injury in an Ex Vivo Rabbit Model of Lung Injury. Ann Thorac Surg 2018;105:950-6.

2. Capdeville R, Buchdunger E, Zimmermann J, et al. Glivec (STI571, imatinib), a rationally developed, targeted anticancer drug. Nat Rev Drug Discov 2002;1:493-502.

3. Chislock EM, Pendergast AM. Abl family kinases regulate endothelial barrier function in vitro and in mice. PLoS One 2013;8:e85231.

4. Aman J, van Bezu J, Damanafshan A, et al. Effective treatment of edema and endothelial barrier dysfunction with imatinib. Circulation 2012;126:2728-38.

5. Stephens RS, Servinsky LE, Rentsendorj O, et al. Protein kinase $\mathrm{G}$ increases antioxidant function in lung microvascular endothelial cells by inhibiting the c-Abl tyrosine kinase. Am J Physiol Cell Physiol 2014;306:C559-69.

6. Ocuin LM, Zeng S, Cavnar MJ, et al. Nilotinib protects the murine liver from ischemia/reperfusion injury. $\mathrm{J}$ Hepatol 2012;57:766-73.

7. Lin $\mathrm{H}$, Chen $\mathrm{M}$, Tian F, et al. $\alpha 1$-Anti-trypsin improves function of porcine donor lungs during ex-vivo lung perfusion. J Heart Lung Transplant 2018;37:656-66.

8. Berger M, Liu M, Uknis ME, et al. Alpha-1-antitrypsin in cell and organ transplantation. Am J Transplant 2018;18:1589-95.

9. An X, Tiwari AK, Sun Y, et al. BCR-ABL tyrosine kinase inhibitors in the treatment of Philadelphia chromosome positive chronic myeloid leukemia: a review. Leuk Res 2010;34:1255-68.

10. Okutani D, Lodyga M, Han B, et al. Src protein tyrosine kinase family and inflammatory responses. Am J Physiol Lung Cell Mol Physiol 2006;291:L129-41.

11. Keshavjee S, Zhang XM, Fischer S, et al. Ischemia reperfusion-induced dynamic changes of protein tyrosine phosphorylation during human lung transplantation. Transplantation 2000;70:525-31.

12. Oyaizu T, Fung S, Shiozaki A, et al. Src tyrosine kinase inhibition protects pulmonary ischemia-reperfusioninduced acute lung injury. Intensive Care Med 2012;38:894-905.

13. Kim H, Zhao J, Zhang Q, et al. $\delta$ V1-1 reduces pulmonary ischemia reperfusion-induced lung injury by inhibiting necrosis and mitochondrial localization of PKC $\delta$ and $\mathrm{p} 53$. Am J Transplant 2016;16:83-98.

14. Sakiyama S, Hamilton J, Han B, et al. Activation of mitogen-activated protein kinases during human lung transplantation. J Heart Lung Transplant 2005;24:2079-85.

15. Anraku M, Cameron MJ, Waddell TK, et al. The impact of human donor lung gene expression profiles on survival after lung transplantation: a case-control study. Am J Transplant 2008;8:2140-8.

16. Kang CH, Cypel M, Anraku M, et al. Transcriptional 
signatures in donor lungs from donation after cardiac death vs. after brain death: a functional pathway analysis. J Heart Lung Transplant 2011;30:289-98.

17. Hu P, Wang X, Haitsma JJ, et al. Microarray meta-analysis identifies acute lung injury biomarkers in donor lungs that predict development of primary graft failure in transplant recipients. PLoS One 2012;7:e45506.

18. Yeung JC, Klement W, Zamel R, et al. Towards donor lung recovery-gene expression changes during ex vivo lung perfusion of human lungs. Am J Transplant 2018;18:1518-26.

19. Barneh F, Jafari M, Mirzaie M. Updates on drugtarget network; facilitating polypharmacology and data integration by growth of DrugBank database. Brief Bioinform 2016;17:1070-80.

20. Fung SY, Oyaizu T, Yang H, et al. The potential of nanoscale combination of self-assembling peptides and amino acids of the Src tyrosine kinase inhibitor in acute

Cite this article as: Huang L, Yang C, Liu M. Intracellular signal transduction pathways as potential drug targets for ischemia-reperfusion injury in lung transplantation. J Thorac Dis 2018;10(Suppl 33):S3965-S3969. doi: 10.21037/ jtd.2018.09.130 lung injury. Biomaterials 2011;32:4000-8.

21. Pacheco S, Kanou T, Fung SY, et al. Formulation of hydrophobic therapeutics with self-assembling peptide and amino acid: a new platform for intravenous drug delivery. $\mathrm{J}$ Control Release 2016;239:211-22.

22. Kim H, Moodley S, and Liu M. TAT cell-penetrating peptide modulates inflammatory response and apoptosis in human lung epithelial cells. Drug Deliv Transl Res 2015;5:275-8.

23. Yang H, Fung SY, Liu M. Programming the cellular uptake of physiologically stable peptide-gold nanoparticle hybrids with single amino acid. Angew Chem Int Ed Engl 2011;50:9643-6.

24. Lee D, Zhao J, Yang H, et al. Effective delivery of a rationally designed intracellular peptide drug with gold nanoparticle-peptide hybrids. Nanoscale 2015;7:12356-60.

25. Mariscal A, Caldarone L, Tikkanen J, et al. Pig lung transplant survival model. Nat Protoc 2018;13:1814-28. 